\title{
Essais
}

ESSAIS

Revue interdisciplinaire d'Humanités

$7 \mid 2015$

Normes communiquées, normes communicantes

\section{La recherche est politique. Pour une approche critique des rapports de domination dans les productions filmiques et audiovisuelles}

Entretien avec Geneviève Sellier

Laetitia Biscarrat

2. OpenEdition

Journals

Édition électronique

URL : http://journals.openedition.org/essais/6456

DOI : 10.4000/essais.6456

ISSN : 2276-0970

Éditeur

École doctorale Montaigne Humanités

Édition imprimée

Date de publication : 1 décembre 2015

Pagination : $112-120$

ISBN : 978-2-9544269-6-9

ISSN : $2417-4211$

Référence électronique

Laetitia Biscarrat, « La recherche est politique. Pour une approche critique des rapports de domination dans les productions filmiques et audiovisuelles », Essais [En ligne], 7 | 2015, mis en ligne le 26 février 2021, consulté le 28 février 2021. URL : http://journals.openedition.org/essais/6456 ; DOI : https:// doi.org/10.4000/essais. 6456 


\section{La recherche est politique}

Pour une approche critique des rapports de domination dans les productions filmiques et audiovisuelles

Entretien avec Geneviève Sellier

\section{Laetitia Biscarrat}

Geneviève Sellier est Professeure en études cinématographiques et audiovisuelles à l'Université Bordeaux Montaigne. Membre de l'Institut Universitaire de France (2008-2013), elle est notamment l'auteure de La Drôle de guerre des sexes du cinéma français 1930-1956 (avec Noël Burch, Nathan Université, 1996), La Nouvelle Vague, un cinéma au masculin singulier (CNRS éditions, 2005), Le cinéma au prisme des rapports de sexe (avec Noël Burch, Vrin, 2009) ou encore Ignorée de tous... sauf du public : quinze ans de fiction télévisée française 1995-2010 (avec Noël Burch, INA/Le Bord de l'eau, 2014).

Laetitia Biscarrat : J'aimerais que nous commencions par présenter votre projet scientifique. Peut-on peut dire qu'il consiste à introduire les Cultural et Gender Studies au sein des études filmiques françaises mais aussi à réhabiliter les productions de la culture populaire comme objets d'études légitimes?

Mon "projet scientifique " a beaucoup évolué depuis 30 ans. Mais l'intérêt pour le cinéma populaire est premier, ou plutôt le souci de prendre en compte la dimension collective et populaire du cinéma, même quand on s'intéresse à un " auteur " particulier, en l'occurrence Grémillon à l'époque de ma thèse. Lutter contre la dimension hagiographique de la cinéphilie en replaçant les films d'auteur dans leur contexte de production et de réception, celui d'une production culturelle qui s'adresse à un large public. Donc, sans rien connaître encore du courant des Cultural Studies, je me suis très tôt située dans le refus de construire le cinéma comme un art d'élite.

Mon intérêt pour les Gender Studies est le résultat de ma situation dominée et marginalisée de femme, que je comparerais volontiers, rétrospectivement, à celle des spectateurs homosexuels décrits par Vito Russo ${ }^{1}:$ le désir de m'identifier

1 Vito Russo, The celluloid closet: homosexuality in the movies, New York, Harper and Row, 1981. 
à des figures féminines valorisantes, m’a fait choisir les films de Grémillon pour faire ma thèse (rétrospectivement, le choix de travailler sur Frontière chinoise (Seven Women) après ma maîtrise sur les derniers westerns de Ford me parait inspiré par la même démarche). Par extension, le constat de la rareté de ces figures féminines valorisantes dans le cinéma français m'a amené à prendre conscience du rôle du cinéma dans la légitimation de la domination masculine et patriarcale. Après la publication de ma thèse sur Grémillon ${ }^{2}$, ma rencontre avec Noël Burch ${ }^{3}$ m’a aidé à accéder aux textes féministes anglophones sur le cinéma, à une époque où ils étaient complètement inconnus en France.

\section{B. : On vous définit parfois comme une historienne du cinéma. Est-ce-que cette étiquette vous semble pertinente ?}

Une grande partie de mon travail prend en compte en effet l'histoire culturelle, c'est à dire la façon dont les films parlent de leur époque, non pas sur le mode du reflet, mais comme une expression de l'imaginaire collectif. D'autre part, dès ma thèse sur Grémillon, j'ai été attentive aux évolutions des mentalités que le cinéma permet de repérer, et leur caractère non linéaire. Cette hypothèse est devenue centrale dans le travail que j'ai commencé avec Noël Burch au début des années 1990, sur le cinéma français des années 1930, 1940 et 1950. En partant des recherches faites par Ginette Vincendeau sur le cinéma populaire des années 30 et sur Gabin ${ }^{4}$, nous avons identifié d'une part des schémas narratifs récurrents correspondant à des périodes relativement courtes, mais surtout nous avons constaté l'extrême sensibilité du cinéma aux grandes secousses politiques de la période, malgré l'apparent éloignement des films de fiction de toute préoccupation directement politique. Le couple incestueux des années trente, le proto-féminisme de tant de films de l'occupation, la misogynie violente de l'après-guerre nous ont amené à faire l'hypothèse d'une part que le cinéma populaire était un observatoire privilégié de l'état des mentalités, et d'autre part qu'il fonctionnait comme un révélateur des non-dits des discours politiques, de ce qui est inavouable dans les rapports sociaux, et en particulier dans les rapports sociaux de sexe, que la société française est particulièrement douée pour occulter.

2 Geneviève Sellier, Jean Grémillon, le cinéma est à vous, Paris, Klincksieck, 1989, rééd. 2012.

3 Né en Californie en 1932, Noël Burch est Professeur des Universités, critique et historien du cinéma. Diplômé de l'IDHEC (Institut des hautes études cinématographiques auquel a succédé La Fémis), il a publié en 2007 De la beauté des latrines (Paris, L'Harmattan). Prenant le contrepied de Théophile Gautier, théoricien de l'art pour l'art, il reproche aux études cinématographiques françaises leur esthétisme élitiste et misogyne auquel il oppose une approche matérialiste ancrée dans ses engagements politiques et féministes.

4 Claude Gauteur et Ginette Vincendeau, Jean Gabin. Anatomie d'un mythe, Paris, Nouveau Monde Éditions, 2006. 
L. B. : Tout au long de votre trajectoire de recherche, vous avez travaillé à déplacer le curseur d'une lecture exclusivement esthétique vers la question du sens et de ses déterminations sociales. En quoi le titre de votre ouvrage de 2005 La Nouvelle Vague : un cinéma au masculin singulier ${ }^{5}$ est-il emblématique de votre démarche ?

Nous avions constaté que notre ouvrage ${ }^{6}$ sur le cinéma populaire des années 30, 40 et 50 avait été largement ignoré par la critique et par nos collègues universitaires en cinéma, malgré la nouveauté objective de notre approche, et notre hypothèse était que le corpus étudié n’intéressait pas la cinéphilie dominante, pour qui le cinéma commence en gros avec la Nouvelle Vague. Par ailleurs, nous avions le désir de voir si notre approche pouvait avoir quelque pertinence pour explorer un cinéma qui se voulait à l'opposé du cinéma populaire, un cinéma qui se voulait personnel, subjectif, plus ou moins transgressif par rapport aux normes narratives et esthétiques du cinéma de genre destiné au grand public.

J'ai donc décidé de m'attaquer, avec les instruments de l'histoire culturelle et des Gender Studies, à ce corpus de films associé à un moment historique, le tournant des années soixante, à l'invention auto-proclamée mais attestée par la presse de l'époque, du cinéma dit "moderne ", et à la revendication, relativement nouvelle, en tout cas depuis le parlant, d'une maitrise individuelle de la création au cinéma.

Le résultat a été très productif scientifiquement mais a vraisemblablement heurté de front les tenants du culte qui s'est instauré en France et ailleurs vis à vis de la Nouvelle Vague, perçu comme le moment inaugural de l'accession du cinéma à la culture d'élite et à l'art moderne (les deux sont étroitement imbriqués). Cet ouvrage, très apprécié comme le précédent par les historien.ne.s et les sociologues féministes, et à l'étranger (les deux sont traduits désormais en anglais), a été littéralement boycotté, encore plus résolument que le précédent, par la critique mais aussi par la plupart des universitaires en cinéma (qui sont souvent les mêmes). Ce boycott en dit beaucoup sur les positions idéologiques et socioculturelles de nos élites, qui tout en se proclamant en général "de gauche » et en revendiquant de s'intéresser à des œuvres d'art "subversives ", refusent obstinément de considérer que les productions artistiques puissent être prises dans des déterminations sociales, qu'elles soient de classe, de genre ou de « race».

5 Geneviève Sellier, La Nouvelle Vague, un cinéma au masculin singulier, Paris, CNRS éditions, coll. Cinéma et Audiovisuel, 2005.

6 Noël Burch et Geneviève Sellier, La Drôle de guerre des sexes du cinéma français 1930-1956, Paris, Nathan Université, 1996. 
L. B. : Les réticences à l'approche Gender ne sont pas le propre des études filmiques. Laurent Le Forestier ${ }^{7}$ souligne la timidité des historiens tandis que Marie-Joseph Bertini et Marlène Coulomb-Gully ont diagnostiqué des facteurs explicatifs de cette frilosité ${ }^{8}$ pour la discipline voisine des Sciences de l'Information et de la Communication. Elles l'expliquent notamment pas l'universalisme républicain, la prégnance de l'ordre symbolique', le soupçon du militantisme ou encore le primat de l'approche empirique et l'interdisciplinarité des SIC. Peut-on identifier les mêmes facteurs pour les études filmiques ? Y a-t-il une spécificité à votre champ disciplinaire?

En partie, il s'agit des mêmes facteurs, mais s'y ajoute le désir de légitimité culturelle d'un média qui a émergé dans le champ de la culture de masse, dans un pays où la culture d'élite est un instrument majeur de distinction sociale (ça ne joue pas aussi fortement aux États-Unis par exemple). Il y a eu la combinaison des analyses marxistes concernant le caractère aliénant de la culture de masse, très influentes dans les années 70 , et le désir de faire reconnaître le cinéma comme art, c'est à dire depuis la fin du XIX ${ }^{\mathrm{e}}$ siècle, en s'adressant à l'élite cultivée, à quoi il faut ajouter le modèle universitaire des études filmiques en France qui est celui des études littéraires, fait d'auteurs et de chefs d'œuvre que l'on analyse inlassablement comme des textes sacrés, et la coupure institutionnalisée entre cinéma et télévision, tant dans la production qu'à l'université : tout cela a contribué à faire des études filmiques un bastion de la résistance aux Cultural Studies et aux Gender Studies en France, cependant que ces approches se développaient de façon exponentielle dans les universités étrangères, anglophones en particulier. Les études filmiques françaises contribuent ainsi à leur propre marginalisation.

Pourtant, les films de fiction ont pour réservoir principal les rapports sociaux de sexe : ce qu'on appelle les histoires d'amour, les histoires de famille mais aussi les histoires d'hommes entre eux (plus rarement les histoires de femmes entre elles) deviennent compréhensibles dans leur diversité avec les instruments des Gender Studies, au lieu d'être considérées comme la répétition éternelle des mêmes intrigues.

7 Laurent Le Forestier, "L'approche "gender" au prisme de l'historiographie du cinéma. Noël Burch et Geneviève Sellier, Le Cinéma au prisme des rapports de sexe ", Mille huit cent quatre-vingt-quinze, $n^{\circ} 60 / 1,2010$, p. 194-201. [En ligne] www.cairn.info/revue-1895-20101-page-194.htm [consulté le 03/05/2015]

8 Marie-Joseph Bertini, "Le Gender Turn, ardente obligation des sciences de l'information et de la communication françaises ", Questions de communication, $\mathrm{n}^{\circ} 15,2009$, p. 155-173. Marlène Coulomb-Gully, "Les sciences de l'information et de la communication : une discipline Gender Blind?", Questions de communication, n 15, 2009, p. 129-153.

9 Notamment la philosophie du droit, la psychanalyse lacanienne et l'anthropologie structuraliste selon Marie-Joseph Bertini, op. cit. 
Par ailleurs, la prise en compte du contexte de réception des films permet d'éviter les surinterprétations, les interprétations délirantes, les anachronismes, mais permet aussi de comprendre l'usage que les contemporains faisaient des films qui leur étaient destinés, pourquoi certains sont des succès, d'autres des fours, pourquoi certains ont fait scandale, et d'autres ont fait consensus, etc. La confrontation entre les films et leur réception initiale permet aussi de faire émerger ce qui est invisible ou tabou pour l'époque.

L. B. : Aujourd'hui le genre s'est fait une large place dans le débat public et scientifique. Des thèses consacrées aux rapports sociaux de sexe au cinéma ont été soutenues, les publications se multiplient et des enseignements en Gender et Cultural Studies sont dispensés dans certaines universités, dont l'Université Bordeaux Montaigne. Finalement, il semblerait que les approches Gender gagnent du terrain. Est-ce-que ce constat vous paraît erroné ou approximatif? Y a-t-il selon vous aujourd'hui davantage de réceptivité à ces approches en France?

Je constate que ces approches intéressent la plupart des étudiant.e.s à qui nos cours s'adressent. Mais les résistances restent vives parmi les universitaires et les modes de recrutement français étant de type féodal et localiste, ce sont les " mandarins " qui l'organisent et qui choisissent souvent leurs propres héritiers, d'autant plus que les universités non parisiennes souffrent d'un déficit de reconnaissance nationale, ce qui freine la circulation des idées et des enseignant.e.s-chercheur.e.s. C'est actuellement un obstacle majeur à la diffusion de ces approches, qui sont en plus accusées d'être des importations américaines, dans un pays qui a fait de l'arrogance culturelle une quasi obligation civique !

L. B. : Quels sont selon vous les chantiers scientifiques émergents en études filmiques autour du genre?

D’abord, il est nécessaire d'explorer, au niveau de la production, les mécanismes qui maintiennent l'existence d'un plafond de verre pour les réalisatrices (depuis plus de 20 ans, le nombre de films réalisés par des femmes en France stagne autour de $20 \%$ ), et suggérer des processus de discrimination positive. D'ailleurs depuis 2014 le $\mathrm{CNC}^{10}$ a, enfin, l'obligation de produire des statistiques sexuées pour les secteurs de la production cinématographique et audiovisuelle.

Concernant les personnages de fiction, nous manquons également de statistiques sexuées. L'article de Mathieu Arbogast ${ }^{11}$ est particulièrement intéressant parce que c'est un travail quantitatif sur le vieillissement des actrices

10 Centre National du Cinéma.

11 Mathieu Arbogast, "De si jeunes femmes... Analyse longitudinale des écarts d'âge et des inégalités de genre dans les séries policières ", Genre en séries : cinéma, télévision, médias, n 1 , 2015, p. 73-99. [En ligne] http://genreenseries.weebly.com/numeacutero-1.html [consulté le 03/05/2015] 
de séries télévisées, ce qui n’avait jamais été réalisé à ce jour. La production de statistiques sexuées par les organismes qui émanent des pouvoirs publics rend visible la domination masculine dans les fictions. Or les créateurs résistent aussi parce qu'ils n'ont aucune conscience de ce phénomène. Ils tendent à considérer qu'ils sont au-delà de toute détermination sociale et que leur liberté est une valeur absolue. Mesurer et quantifier la réalité de ce qu'ils produisent peut être un levier d'action pour ouvrir le débat. Si on ne peut favoriser des projets au motif que les femmes y seraient plus visibles, critère qui oblitèrerait les questions de qualité, en revanche produire et diffuser des statistiques sexuées sur les univers fictionnels serait une façon de s'emparer de cette question qui pour l'instant demeure totalement taboue.

Le chantier principal, que nous avons à peine entamé, reste celui d'une approche critique des rapports de domination genrée dans les productions filmiques et audiovisuelles, et le récent scandale concernant le harcèlement dont a été victime une blogueuse qui critiquait le machisme des jeux vidéo ${ }^{12}$, montre que ces phénomènes perdurent à un niveau inquiétant en France, y compris dans la jeunesse (et comment en serait-il autrement tant qu'il n'y aura aucune éducation à l'égalité fille/garçon ?). Le fait que les Gender Studies soient aussi un enjeu civique et démocratique, leur donne une valeur supplémentaire, sauf à considérer que la recherche n'est valable que lorsqu'elle n’a aucun impact sur la société !

Cette approche critique des représentations genrées dans les productions filmiques et audiovisuelles est pourtant loin d'être évidente. En effet, elle est malheureusement moins valorisée que tout ce qu'on met sous le vocable des études Queer. L'attention aux groupes minoritaires, tout à fait souhaitable, a tendance à être mise en œuvre d'une façon non-critique. Il s'agit alors de valoriser des représentations minoritaires en passant sous silence la critique des rapports de domination majoritaires. En effet, l'homosexualité n'est pas en soi subversive. Elle devient subversive lorsqu'elle s'accompagne d'une prise de conscience des processus de domination et marginalisation dont elle est l'objet et qu'elle est capable de les penser socialement. Dans les milieux contemporains de la culture, les effets de la levée de ce tabou sont surprenants : dans un certain nombre de lieux et de disciplines culturelles, l'exclusion des femmes s'en trouve accentuée tout en étant masquée par une prétention à la subversion. C'est notamment le cas de la danse contemporaine, comme l'a très bien montré Hélène Marquié ${ }^{13}$. Le jeu des chorégraphes masculins avec les stéréotypes de la féminité participe de l'exclusion des femmes de leur

12 Voir les menaces subies aux États-Unis par Anita Sarkeesian (Feminist Frequency) et en France par Mar_Lard.

13 Hélène Marquié, "Jeux de genre(s) dans la danse contemporaine ", Journal des anthropologues, Association française des anthropologues, $n^{\circ}$ 124-125, 2011, p. 287-309. [En ligne] http://jda. revues.org/5853 [consulté le 04/05/2015] 
scène. Dès lors, je ne considère pas les études Queer en soi comme des études critiques. Dans la pratique, elles peuvent être une niche pour une culture élitiste, misogyne et narcissique.

L. B. : Ne doit-on pas voir là un effet du backlash antiféministe ? La société française se définit comme égalitariste et rend difficilement questionnable la reproduction des discriminations de genre tandis qu'elle implique d'y répondre individuellement ${ }^{14}$.

Il s'agit à la fois d'un effet de l'individualisation des pratiques et des comportements et d'une tendance très contemporaine de la culture à fonctionner en niches culturelles. En conséquence, les recherches sur les secteurs de productions culturelles minoritaires et marginales gagnent en visibilité, à l'Université tout du moins, alors que le terrain majoritaire des productions de la culture de masse n'est pas investi. Ces productions n'attirent guère les jeunes universitaires qui y voient beaucoup de conventions, ce qui est vrai et faux, comme Noël Burch et moi l'avons montré dans notre ouvrage sur les téléfilms unitaires ${ }^{15}$.

Un des obstacles objectifs aujourd'hui à une approche critique systématique des productions filmiques et audiovisuelles françaises est lié au développement chez les jeunes, et notamment chez les jeunes chercheur.e.s, d'une culture de niche qui passe soit par le web, soit par les séries américaines. Une fois de plus la production culturelle française, quand elle n'est pas d'auteur ou expérimentale, disparaît.

Or, à mon sens les universitaires ont la responsabilité de s'emparer de ces corpus : ce qui est en jeu c'est l'avenir de notre société tout simplement. Se dirige-t-on vers une société où les écarts sont de plus en plus grands entre une majorité qui n'a ni pouvoir ni influence et une élite cultivée qui fonctionne de manière ghettoïsée ? On ne saurait prétendre à être une société démocratique si on est dans une situation d'apartheid culturel, ce qui est un horizon possible de la société française.

Le contre-exemple de la société américaine est de ce point de vue-là frappant. Toute inégalitaire qu'elle soit, celle-ci est culturellement moins clivée que la société française. Ainsi les séries télévisées, pas seulement celles des chaînes du câble mais aussi celles des networks, développent une inventivité narrative et également sociale dont nous n'avons pas l'équivalent. D'abord, les moyens mis en œuvre diffèrent ; mais surtout la perspective de créer pour la télévision et plus particulièrement pour les séries, sauf si c'est pour Arte,

14 Voir Éric Macé, «Le piège de la "cause des femmes". Éléments pour un mouvement antisexiste post-féministe ", Cosmopolitiques, n ${ }^{\circ} 4,2002$, p. 84-103. [En ligne] http://www.cosmopolitiques.com/sites/default/files/Mace_Post_feminisme.pdf [consulté le 03/05/2015]

15 Noël Burch et Geneviève Sellier, Ignorée de tous... sauf du public : quinze ans de fiction télévisée française 1995-2010, Paris, INA/La Bord de l'eau, 2014. 
apparait à l'élite cultivée comme une "humiliation absolue ». Ces spécificités françaises sont à mon sens un obstacle au développement d'une approche critique des rapports de genre dans les productions filmiques et audiovisuelles.

L. B. : Teresa de Lauretis écrit que «la théorie (un terme générique valant pour tout discours théorique dont l'objectif est d'essayer d'expliquer un objet particulier avec pour effet de construire cet objet dans un champ de signification qui devient son propre domaine de savoir, ce domaine étant souvent appelé "discipline") est une technologie de genre ${ }^{16}$. Reconnaître que nos pratiques de recherche participent de la re-production du genre nous incite dès lors à entreprendre une démarche réflexive et critique sur nos positionnements et nos méthodologies. Comment mettez-vous en œuvre ce travail dans le champ des études cinématographiques et audiovisuelles?

Nous avons besoin constamment d'adopter une démarche réflexive afin de ne pas essentialiser nos positions. La resubstantivation des identités de genre est un écueil des études de genre, même lorsqu'on se définit comme matérialiste. Il ne s'agit pas néanmoins de pécher par idéalisme. Nous vivons dans une société dans laquelle ces catégories sont totalement structurantes. En identifiant un bébé à la naissance d'abord par son sexe, ce qui reste déterminant jusqu'à la mort, la société nous structure totalement dans nos identités genrées.

Contrairement à un positionnement qui poserait comme possible de s'abstraire des identités de genre, il faut à mon sens admettre que nous sommes pris dans ces rapports. La seule chose qu'on peut faire, et que finalement très peu d'entre nous font, consiste à adopter constamment un regard critique. Est-ce-que je fais ceci parce que j'accepte les stéréotypes genrés ou est-ce-que je fais cela parce que j'ai décidé de le faire et que cela correspond à ce que je veux faire ? Il est important de donner au plus grand nombre les moyens d'une réflexion critique sur les stéréotypes genrés dans lesquels chacun.e est enfermé.e. Au-delà de la question des études cinématographiques, la reconduction des assignations genrées est saisissante. L'importance du travail critique sur les déterminismes sociaux dans lesquels nous sommes construit.e.s me paraît la chose la plus utile que nous puissions faire.

L. B. : Le projet des Gender Studies consiste alors à partir de sa propre subjectivité, en faisant le deuil de l'objectivité et de ses implications positivistes, tout autant qu'à reconnaître la dimension politique des approches critiques. D'un point de vue méthodologique, peut-on utiliser des outils « classiques" pour mettre en ouvre ce projet?

16 Teresa de Lauretis, Théorie queer et cultures populaires. De Foucault à Cronenberg, Paris, La Dispute, 2007, p. 77. 
Oui tout à fait, "le personnel est politique " et "le scientifique est politique»! Dans les approches genrées du cinéma, l'instrument théorique qui a été privilégié notamment par les Américains est la psychanalyse. Je pense qu'il s'agit d'une impasse. Cela a été important pour mettre au jour l'importance des sphères préconscientes dans la construction des identités genrées et pour relativiser les "intentions de l'auteur ». Les travaux de Christian Metz ou de Laura Mulvey sont très utiles. Nous souffrons aujourd'hui d'un déficit en termes d'outils méthodologiques et théoriques. De ce point de vue-là il serait intéressant de créer une synergie entre les Études Cinématographiques et Audiovisuelles et les Sciences de l'Information et de la Communication.

Ce travail considérable concerne tout le monde. La responsabilité sociale des universitaires est absolument engagée à partir du moment où ils/elles sont des citoyen.ne.s. Cette responsabilité sociale consiste à construire des instruments pour une approche critique des artefacts culturels quels qu'ils soient. Ces instruments doivent être suffisamment rigoureux pour qu'ils puissent être transmis et servir la bataille politique de l'égalité, qui est mon horizon, ou plutôt de cette bataille politique qui consiste à permettre aux femmes aussi de se construire comme des êtres humains à part entière, comme des sujets de leur propre destin.

\section{Entretien réalisé par Laetitia Biscarrat \\ Université de Saint-Étienne laetitiabiscarrat@hotmail.com}

NDRL : Entretien réalisé le 13 avril 2015 à l'Université Bordeaux Montaigne. 